\title{
Weak Interaction and the Nature of Virtual Particles
}

\author{
Vladimir Konushko \\ Protvino, Moscow Region, Russia \\ E-mail: konushko@mail.ru \\ Received December 10, 2010; revised January 18, 2011; accepted February 20, 2011
}

\begin{abstract}
The granular structure of space, the nature of virtual particles and the unity of physical interactions are under study. The mass of an elementary cell in the Universe is determined, as a whole, with the physical meaning of the renormalization procedure changing in this case.
\end{abstract}

Keywords: Weak Interaction

\section{Particle Motion in Space}

To derive a theoretical expression for the dependence of black-body radiation intensity on wavelength and temperature, Planck made a special suggestion [1] which means that an oscillator making a free oscillation with the frequency may gain or release energy in portions whose value:

$$
E=h v .
$$

A little later Einstein suggested that the electromagnetic radiation in a cavity is corpuscular, that is, consist of quanta with their energy $h v$.

If we consider just one photon, the presence of $v$ frequency in this expression (for visible light $v=10^{13} \mathrm{l} / \mathrm{s}$ ) is rather unusual. To increase our surprise let us consider another physical phenomenon - sound propagation. The note "la" is produced by tuning fork legs oscillating with $v=430 \mathrm{~Hz}$. It means that eventually our tympanum (ear-drum) can feel 430 blows of air molecules per sound. If the photon frequency, however, $v=10^{13} \mathrm{~Hz}$, our eye can, all the same, catch only one photon, and not $10^{13}$. Thus, the characteristic of one photon must contain something of unity, the only thing that characterizes its one oscillation.

Schrodinger wrote that a science in which the Universe is just described but does not explain why it is made so and not otherwise is an ungrounded theory.

It is only the period $T$ that is unitary for a photon. Besides, it is not $\mathrm{h}$, but $\hbar=h / 2 \pi$ that enters into all the quantum characteristics of microcosm.

Let us transform the Plank formula thus:

$$
E=\hbar \omega=\hbar 2 \pi / T=\hbar(T / 2 \pi)=\hbar / t_{r}
$$

The quantity $t_{r}=T / 2 \pi$ in the vibration theory means the time in which a body oscillating with the con- stant frequency $\omega$ covers an angle of one radian. And what have the constant $\omega$ and an angle of one radian to do with photon?

It is of interest that the up-down oscillations of a weight on a string are related to the circular motion of a point. It can be checked by the following experiment. Arc-discharge lamp light projects onto a screen the shadows of a ball pressed on a disc and a vertically oscillating weight moving alongside. If we force the weight to oscillate in good time and from a wanted place and then carefully choose the velocity of travel of the disc so that the frequencies of their motions coincide, the shadows on the screen will accurately follow each other (Figure 1); the oscillations of the weight and the shadow of the ball moving in the circle with a constant velocity obey one and the same low!

It is intriguing that nature describes two quite opposite physical phenomena with the same equation: uniform motion and broken pulsating motion.

Maxwell was astonished by the similarity of the equa-

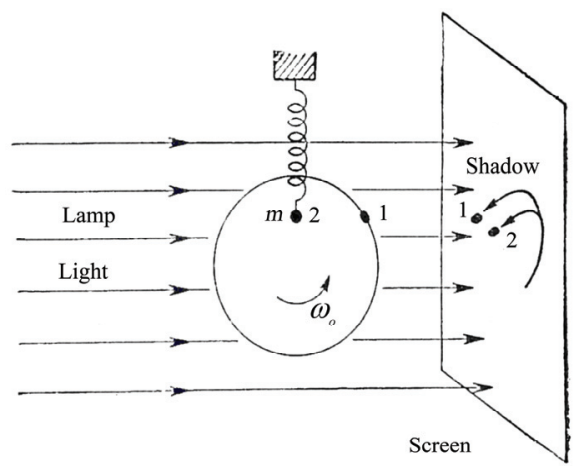

Figure 1. Equivalence of simple harmonic motion and uniform circular motion. 
tions describing electromagnetic oscillations and pendular oscillations.

The wonderful analogy unravels the secrets of the presence of the $\omega$ frequency in Planck formula as well as the character of photon motion and answers the question which Einstein used to ask himself in his youth: what would we see if we ran alongside a photon?

To support our arguments we turn mentally to the experiment and, while moving at the velocity of light, we shall observe the motion of the photon. Since granular space is uniform and isotropic, the deformation of cells lengthwise will be equal to transverse deformation. The latter spares us the false idea that only solids possess transverse elasticity and unravel the secret - how the transverse polarization which surprised very much $\mathrm{T}$. Young can occur in empty space.

Furthermore, passing from one elementary cell and moving both lengthwise and crosswise, deformation will cover the cells lying in a sector of one steradian because the process occurs the three - dimensional space but not in a plane.

A photon has energy and, hence, it possesses a mass. Besides, photon transfer is the motion of elastic deformations of cells and not the transfer of cells themselves in contrast to the wind carrying air masses.

A deformation wave brings about denseness in the cell distribution, but it does not pull the cells after itself and passes from cells to cells forming a cluster of new cells in other place. This is a characteristic feature of any wave process.

At a certain distance referred to as the wavelength $\lambda$ the reverse process begins, when the deformation is reduced to one cell. This surprising pulsating process differs drastically from the motion of a sea wave since, while moving alongside this wave, we could observe one and the same profile - a hump.

The motion of any elementary particle is quite similar to that of a photon. The mass carried by kinetic energy moves ahead of the particle and deforms the cells of the space in the same pulsating manner but at an angel smaller than one steradian since the velocity of the particle is less than that of light beyond material media. The particle itself, however, can never become a wave; an electron, for example, remains individual even with $E \approx 10^{19} \mathrm{GeV}$. It obtains wave functions due to the pulsating motion of the matter carried by kinetic energy which reveals the mystery of "centaur", i.e., waveparticle. This new look on this space structure made us forget about the "centaur" and introduce a new object: "a rider on a horse".

The "horse" means the substance (the mass), carried by kinetic energy moving ahead of the particle, the "rider" means itself.
At first glance the slight difference between the pulsating motion of particles and photons and the motion of a sea wave plays a large role of revealing the physical meaning of such phenomena as weak interaction, tunnel effect, the passage of electron through two slits, the causes of probability manifestation in many physical processes, the dependence of the wave function phase on distance - $\varphi(x)$ and others we are going to consider in the further articles.

It is of interest to know what the photon wavelength $\lambda$ depends on and why the deformation of cells in this case changes the nature of their motion: from expansion to compression. In space there is just one reason for it: the wave edge cells from at this instant a surface with its envelope being spherical. The deformation of the cells in such a layer will be only tangential and there will be no reason for further expansion.

At this instruct the area of the layer filled with deformed cells is $\lambda^{2}$, so it covers an angel of one steradian. But the untangling of the physical meaning of the constant fine structure $a=e^{2} / \hbar c \approx 1 / 137$ is more impressive.

As it has been mentioned repeatedly, it is a football made of segments of positive curvature (positive charge) or negative curvature (negative charge) roughly reminds us of a charged particle. The area of such a segment in this case makes up $a \approx 1 / 137$ of the area equal to $\lambda^{2}$, whereas the angel covered by one segment makes up $\Delta \Omega \approx 1 / 137$ of an angle of one steradian. Multiplying $1 / a \approx 137$ by $4 \pi$ we get the number of segments housed on the surface of a charged particles, $N_{s}=1722$, which is another puzzling number.

In the above mental experiment we could have observed torsional deformation of elementary particles associated by us with a magnetic field and with a spin in case of particles at rest. In more detail we shall speak about the spin in other article.

Now the Plank formula can be expressed as:

$$
E=\frac{\hbar}{t_{r}},
$$

where $t_{r}=T / 2 \pi$ is the time of cell deformation propagation at the distance $\lambda$ at an angel of the steradian.

Thus, the Plank formula gains the quantity $\omega$ and the number $\pi$ which, at first glance, have nothing to do with translational motion.

\section{Nature of Virtual Particles}

Let us consider now a physical process where a "virtual" photon, and not a real one, appears. An example exists in the process $e^{-} p$-distance:

$$
e^{-}+p \rightarrow e^{-}+p
$$


In terms of classical physics we can say that on electron and a proton interact because the electron "feels" the electromagnetic field of the proton which affects the motion of the electron. In the quantum field theory, however, the electromagnetic field is quantized and becomes similar to particle - photon and then we say that the electron and the proton "exchange" a photon. The process of this exchange is illustrated in Figure 2 using the Feynman diagram.

A photon is "virtual" because it is emitted and absorbed in such a short time that, according to the uncertainty principle, cannot be detected.

The considered motion of a real photon and any elementary particle saves us from a touch of mysticism connected with the concept "virtual".

The real matter (mass) carried by kinetic energy serves as a virtual photon, a virtual $\mathrm{W}$-boson, a virtual gluon, etc. depending on the type of interaction. There is not even a miniscule touch of "virtuality" in it: real matter, i.e. a real object moves in real material space.

"Any physical ideas need common sense in their realization because they are not purely mathematical or abstract ideas." - Feynman [2].

Appreciating the tradition and the accepted terminology we call, as before, this object virtual. It has not got a closed shell (surface), and therefore the equality $E^{2}-\vec{p}^{2} c^{2}=m^{2} c^{4}$ is not fulfilled.

From this a purely mathematical result can be derived: the left term may be either above zero or below zero, but there is no mysticism in this case. Besides, unlike the case of a real photon, the deformation of cells in a virtual object develops, as we have already noted, into an angel smaller than one steradian. Their common feature is pulsating nature of motion which is not transient for both microphenomena and microcosm.

The Geisenberg's inequalities, however, only belong to the variation of characteristics of elementary particles, as it follows from the works of Geisenberg, and result in a spread and not uncertainty in measuring one or another value: $\Delta p, \Delta x, \Delta E$, etc. So almost a century later after these works were published, the new concept of space, interaction and motion developed in the theory of granular space enables us to speak only about Geisenberg ine-

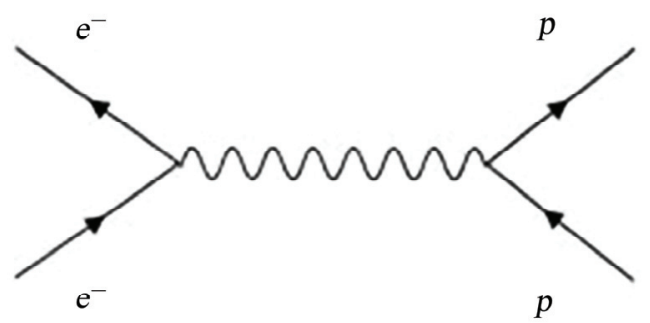

Figure 2. Feynman diagram of electron-proton scattering caused by photon exchange. qualities. "If a mouse looks at the Universe, does the Universe change because of this?" asked Einstein who did not assume even the slightest uncertainty or indeterminism in microcosm.

"To speak about indeterminism means indulging in mental depravity," - the French physicist P. Langevin expressed himself so sharply.

\section{Weak Interaction and Mystery of W - and Z-Bosons}

\subsection{Elementary Granule Mass}

Every new prospect opened before the granular space theory derives from three fundamental assertions [3]:

1) The Planck length $L^{*}=\sqrt{2 G \hbar / c^{3}}$ is a dimension of a three - dimensional elementary granule of space. It is such granules that from space at their deepest level [4].

2) The dimension of an elementary particle is equal (leptons) or a little than its Compton length:

$$
r \geq \hbar / m c \text {. }
$$

3) The quantity $\varepsilon=v^{2} / c^{2}$ is the relative deformation of elementary granules.

These postulates open basically new ways of investigation of elementary particles in the Universe on the whole.

Let us count again the number of elementary granules which form the surface of an electron:

$$
N_{\text {cell }}=\frac{4 \pi r_{e}^{2}}{\left(L^{*}\right)^{2}}=\frac{4 \pi \hbar c}{G m_{e}{ }^{2}} \approx 7,32 \cdot 10^{45}
$$

When solving the problem of large number in our first work "Granular space and the problem of large numbers", where we considered the ratio of Coulomb force to gravity for all elementary particles which is equal for the electron:

$$
\left(\frac{F_{k}}{F_{n}}\right)_{e}=4,17 \cdot 10^{42},
$$

We found that if in a mental experiment two particles are pressed lightly to each other, till their surfaces determined by the Compton length $\lambda$ come in contact, only the matter falling on one elementary cell of the surface layer will be involved in gravitational interaction. The surface of a charged particle, for example, an electron consists of clusters, each of them having $4,17 \cdot 10^{42}$ cells.

Nature solves the problem of large numbers in a simple and original way: the electron surface is covered with clusters of negative curvature, the positron surface - the clusters of positive curvature, and in an ultimate case (contact) the electron and the positron touch one another with a surface containing a great number of cells. In more detail the essence of electric charge will be consid- 
ered in another article.

Besides, the smallest change of space in one elementary cell: there is no matter of half a cell, a third of a cell, etc.

Hence, to create an electron space spends the matter of the cells placed on the surface of a particle, with regard to the fact that the "body" of any particle is made of cells of the space itself.

One hundred and fifty years ago the English mathematician B. Clifford stated oracularly that matter is clusters of space, i.e., peculiar curvature peaks against the background of flat space.

When two particles move apart, it results in two factors: a decrease in space cell deformation and a relative decrease in cluster area which results in a force-distance dependence:

$$
F \sim \frac{1}{r^{2}} .
$$

To form a particle, additional matter is needed, i.e., mass of a particle at rest. This mass has to deform (to press the inner and the other cells of the particle "body" in order to be quartered among the existing space cells. As the particle mass grows, the deformation of inner cells increases too, and beginning with the $\pi$-meson mass where it is well for space to form clusters inside a particle a structure appears. These clusters are termed to as quarks, and the continuous deformation of the cells placed between clusters enabled us to introduce the concept of gluons. Furthermore, the larger the particle mass, the more is the cell deformation and the smaller the particle size. Hence, nature dictates a rigid dependence of the size of a particle and its mass at rest $m_{0}$. The matter of an electron and muon is not enough for the inner deformed cells to form clusters (quarks), so when interacting electrons, muons and quarks behave like point particles and so give birth to a false notion that their size $r<10^{-17} \mathrm{~cm}$.

The quark confinement becomes quite transparent. In space free of cell deformation they cannot be formed and cannot exist beyond a particle.

Long ago many physicists suspected that a combination of Einstein's theory of gravity with quantum mechanics may terminate in giving up the idealization of continuous space and in detecting space "atoms".

At present more and more physicists conclude that space has a granular structure and presets an absolute system of coordinates $[5,6]$.

Could Einstein think about it when in 1954, a year before his death, wrote to his close friend M. Becco: "It is not excluded that physics cannot be based only on the concept of field, i.e. on continuous structures. But I this case nothing will remain of my castle in the air, even the theory of gravity as well as the whole contemporary physics".

This unwarranted pessimism is completely invalidated by the theory of granular space. Einstein's theory of gravity is valid because the tensor $R_{i k}$ and the scalar curvature $R$, which enter the Einstein equation, describe the deformation of elementary space particles caused by massive bodies and the cell deformation, in its turn, makes the former flat surface curved which can be clearly observed on a simple model of honeycombs. In our separate article we shall study Lorentzian transformations and Einstein's theory of gravity.

An elementary cell is a quantum of space but, since space is quantized, the concept of "physical field" does not lose its fundamental importance. In the 30 -s of XIX century Faraday introduced the concept "field" and in 1852 formulated the concept of electromagnetic field. This idea, in Einstein's opinion, was the most important discovery since Newton's times.

It is deformed space cells that make up the conception of physical field which is as material as elementary particles. All the types of physical field (Colombian, magnetic, nuclear and other) are deformed cells which differ from each other by the degree of deformation and their structure.

We also want to stress the fact that the field itself is characterized by mass [7].

Einstein should not have worried about the disappearance of field in discrete space. On the contrary, now we can visualize it by enlarging on elementary cell in a mental experiment. In our studies both particles and fields are part of space.

It is discrete space that is responsible for quantization of the energy, the impulse, the momentum and spin of elementary particles. The essence of quantization is the formation of clusters, i.e., huge ensembles consisting of individual elementary cells of space.

The quality of additional cells, whose matter forms the mass of particle at rest, with the number of cells on the electron surface is in a unique peculiarity of only this particle. To form other particles space spends more matter of cells than placed on their surface.

By concretizing the concept of "elementary cell" and using (1) we can find the amount of matter (mass) which space spends to produce one elementary cell:

$$
m_{\text {cell }}=\frac{m_{e}}{N_{\text {cell }}}=\frac{G m_{e}{ }^{3}}{4 \pi \hbar c} \cong 1,3 \cdot 10^{-73} \mathrm{~g}
$$

To confirm energy falling on one elementary cell of electron surface is:

$$
E_{\text {cell }}=\frac{m_{e} c^{2}}{N_{\text {cell }}} \cong 0,7 \cdot 10^{-40} \mathrm{eV}
$$

To confirm and adjust this value of $E_{\text {cell }}$, we can turn to a physical phenomenon which, at first sight, has noth- 
ing to do with neither an elementary cell nor an electron. This phenomenon is radioactive decay of nuclei.

In the history of science the number of scientific discoveries made by accident is not large as many may think. But beyond question is the accidental character of one of the greatest discoveries made by physicists in the 20 th century - radioactivity.

In experiments $\alpha$-radioactivity has been discovered for nuclei occurring in nature, beginning with $z=83$. Lead ${ }_{82} \mathrm{~Pb}^{208}$ is a stable element. The nuclei ${ }_{83} \mathrm{Bi}^{209}$ and ${ }_{92} \mathrm{U}^{235}$ have the longest half-life period $T_{1 / 2}$. Alongside $a$-decay, the nuclei ${ }_{92} \mathrm{U}^{235}$ undergo spontaneous fission. But the most surprising thing is that the mean lifetime of these nuclei is the same, $\tau \approx 3 \cdot 10^{17}$ years. We don't consider double $\beta$-decay here because there are two nucleons participating in this process.

Sing the Geizenberg inequality (not uncertainty) for spread of energy we have

$$
\Delta E=\frac{\hbar}{\tau} \cong 0,7 \cdot 10^{-40} \mathrm{eV}
$$

The agreement between (3) and (4) stupefies us: the spread of energy is equal to the energy (mass) of one elementary cell. Both the order of power (40) and the significant figure $(0,7)$ coincide, too. As stated above, an elementary cell is a "small change" of the Universe. So, the statement of the mathematician E. Kronecker sounds as a prophecy: "Natural numbers are created by God, all the rest is handiwork of man." No doubt, the role of the Creator is player by nature itself which not know any other numbers but natural ones.

\subsection{Do Protons Die?}

The fact that the longest half-life periods coincide immediately for several nuclei shows that there is not a higher half-period in nature. Besides, as mentioned above, the spread of energy cannot be less than the energy of one cell.

The proton lifetime is known to exceed $\tau_{p}>10^{33}$ years. In this case the spread of energy is $\Delta E_{p}<10^{-16} \cdot E_{\text {cell }}$, which is completely excluded. We have proved the absolute stability of proton. This conclusion cannot be overestimated since it enables us to hope that the Universe, stars and planets will always exist and, hence, wise life. Below we are going to consider the possibility of decay of a proton with the participation of a heavy calibrated X-boson.

Dozens of laboratories al over the world compete in experiments on proton decay because the stakes are too high. But our studies show that they are all doomed to failure.

The absence of proton decay casts a shadow on the existence of heave $\mathrm{X}$ - and $\mathrm{Y}$-bosons in nature and on the hypothesis of great synthesis.

\subsection{Is Motion Possible in "Boiling" Vacuum?}

Going on with our studies into an elementary space particle we must determine its density as it is done in macroscopic physics:

$$
\rho_{\text {cell }}=\frac{3 m_{\text {cell }}}{4 \pi\left(L^{*}\right)^{3}}=1,1 \cdot 10^{25} \mathrm{~g} / \mathrm{cm}^{3}
$$

It should be noted that using the Gödel theorem of incompleteness we can judge the degree of materiality of "boiling vacuum" where at every instant and at any point of space various pairs of "virtual" particles are formed and disappear. According to this theorem, in any formal and rather complicated axiomatized system there are always meaningful suggestions which can be expressed in the language of this system, but it is impossible to prove their truthfulness of falsity in this language.

To estimate the viability of "boiling" vacuum we must perform the following real experiment, not mental. Let us consider an energetic electron moving in this vacuum. Various pairs of charged "virtual" particles will always be born on its way for a short instant. Since the electromagnetic interaction cross-section is large enough, the electron collides with such particles and passes them some energy thus making them real. As a result, it instantly stops and we can observe the birth of additional real particles in vacuum.

The conclusion of this situation is simply stunning: any motion is impossible in "boiling" vacuum!

It must be said at once, we did not observe anything of the kind in the experiment. Below, as well as in a separate article, we shall touch upon virtual particles.

And now let's turn to the most unexplained phenomenon - weak interaction.

\subsection{Mystery of W-Boson}

The probabilities of all weak reactions and weak decays of elementary particles are proportional to the squared Femi constant $G_{F}{ }^{2}$. The muon lifetime can be expressed by $G_{F}$ and its mass $m_{\mu}$, with the electron mass neglected, in this way:

$$
\frac{1}{\tau_{\mu}}=\frac{G_{F}{ }^{2} \cdot m_{\mu}{ }^{5} \cdot c^{4}}{192 \pi^{3} \cdot \hbar^{7}}
$$

In the standard theory of Weinberg-Salam $[8,9] G_{F}$ is related to the dimensionless coupling constant of weak interaction $g_{w}$

$$
\frac{G_{F}}{\sqrt{2}}=\frac{g_{w}{ }^{2}}{8 M_{w}^{2}},
$$

where $M_{w}$ is the mass of W-boson, $M_{w} \cong 83$ 
$\mathrm{GeV} / \mathrm{c}^{2}$. To reveal the physical meaning of (6) we regroup the factors in it:

$$
\frac{1}{\tau_{\mu}}=\frac{\text { const }\left(\frac{g_{w}{ }^{2}}{\hbar c}\right)^{2} \cdot \frac{m_{\mu} c^{2}}{\left(\hbar / m_{\mu} c\right)^{3}} \cdot \frac{m_{\mu} c^{2}}{\hbar}}{\frac{M_{w} c^{2}}{\left(\hbar / M_{w} c\right)^{3}}}
$$

According to the standard theory of weak electric interaction, all the interactions between charged currents are realized through exchange of virtual W-bosons born by "boiling" vacuum for a short time (Figure 3). The first stage of muon decay includes the transformation of a muon into a muon neutrino $v_{\mu}$ with simultaneous emission of a heavy virtual W-boson. The second stage is the decomposition of a virtual particle $W$ to an electron $e$ and an electron antineutrino $v_{e}$.

But the Formula (7) contains the mass of a real Wboson to the fourth power rather than a virtual one! How has the colossal mass of a real W-boson to do with this reaction which is characterized by small energy release: $m_{\mu} c^{2} \approx 105,6 \mathrm{MeV}$ ?

The situation is aggravated by the fact that the law of energy conversation in this reaction (as well as in all other processes) is met impeccable: the energy of initial state is accurately equal to that of final state.

It is known at present that it is reasonable to present all natural forces as a result of exchange of virtual particles for which the stable law of energy conservation may be violated. This serious accusation to space was brought by $\mathrm{N}$. Bohr who turned the simple Geisenberg inequality:

$$
\Delta E \cdot \Delta t \geq \hbar
$$

To the uncertainty principle with the use of it he wanted to explain the stability of atom. This problem will be considered in more detal in a separate article.

The quantity $m_{\mu} c^{2} /\left(\hbar / m_{\mu} c\right)^{3}$ in (7) is the density of the energy released in the process of muon decay without regard for a small electron mass.

In our first paper we started that the dimensions of all leptons are completely determined by the Compton length:

$$
r \cong \lambda=\frac{\hbar}{m c} .
$$

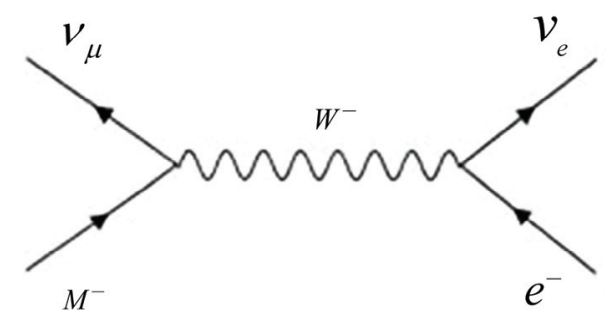

Figure 3. Scheme of decay of muon $\mu^{-}$.
As for the electron dimensions, Yukava and Landau held the same viewpoint.

In our articles we repeatedly paid attention to the false concept of an electron as a point particle whose dimension $r_{e}<10^{-17} \mathrm{~cm}$. In the material Universe there are not any point objects and there cannot be. This must be understood that a particle has no structure, i.e., cannot be divided into components. The number $10^{-17} \mathrm{~cm}$ means that, if there are any structural formations inside on electron, they are less than this number which has nothing to do wit the size of the electron itself.

The hypothesis of point electron solves just strictly specialized tasks and does not solve any of a broad range of fundamental problems.

With this comment we part for ever with the classical infinity of intrinsic electron energy which appears if the electron dimension $r_{e} \rightarrow 0$. A little later we shall part with the quantum infinity which leads to renormalization.

Consequently, the dimension of W-boson

$$
r_{w} \cong \lambda_{w}=\frac{\hbar}{M_{w} c}
$$

and the factor $M_{w} c^{2} /\left(\hbar / M_{w} c\right)^{3}$ is equal to the rest energy flux of W-boson (real).

The relation between these two quantities indicates convincingly that the energy released in decay must be grouped into a dense lump, the density of which must be equal to the analogical quantity of W-boson, in order to destroy the dense layer of muon cells.

It cannot be doubted that it is this dense energy clusters that can be called "virtual" W-boson in the up-todate theory. This boson, it its turn, gives birth to "boiling" vacuum.

This relation between the two quantities has another advantage since it is quite probable that the particle will decay, and so we can visualize a probability during collisions and decays of elementary particles. Despite the fact that the formula has a huge mass of real W-boson this is just its "shadow", i.e., the density of its mass.

It must be stressed again that the pulsing character of motion of the substance cluster released at decays and collisions of particles plays a leading part.

There is a simple analogy to particle collision: the bombarding particle is a "hammer", the bombarding particle is an "anvil" and the matter carried by kinetic energy is a half-finished product. Note that, from the viewpoint of the up-to-date field theory, interaction is carried by quantum-particles of the field involved. Clearly it is energy, momentum and, hence, mass that are transferred with a quantum.

All particles are formed from a half-finished product with its matter used just to make the surface layer of new particles; the "body" of a new particle will be made of 
space cells. Space in this case acts as a "creditor."

Going on with this analogy we can represent fundamental interactions in the form of keys, and even though these keys are different (interaction constants differ in intensity), all of them are made of one and the same half-finished material, i.e., the matter transferred by the kinetic energy of a bombarding particle. That is why a virtual photon, a virtual $\mathrm{W}$-boson and a gluon possess a spin, equal to unity, and odd parity.

Now we are sure that a simple regrouping of factors can greatly simplify the formula and do even more - to get inside the physical nature of the process.

Of exceptional mystery is the fact that only weak interaction is able to rebuild the particle structure or to change the quark ordour, as well as the participation of the characteristic (substance density) intrinsic in a real $\mathrm{W}$-boson in this process.

An elementary particle can help us to solve this problem again. Previously, we determined its macroscopic density: $\rho_{\text {cell }} \cong 1,1 \cdot 10^{25} \mathrm{~g} / \mathrm{cm}^{3}$.

It is of interest to know if there is a particle whose substance density is equal to that of an elementary cell. As well as before,

$$
r \cong \lambda=\frac{\hbar}{m c},
$$

then

$$
V \sim r^{3}=\left(\frac{\hbar}{m c}\right)^{3}, m=\rho_{\text {cell }} V
$$

and

$$
m \cong \sqrt[4]{\frac{\hbar^{3} \cdot \rho_{\text {cell }}}{c^{3}}} \cong 83 \mathrm{GeV} / \mathrm{c}^{2}
$$

Taking into account our simplified approach and the fact we have not used any fitting parameters we cannot help being stunned by this result! This mass $m$ is equal to the mass of W-boson:

$$
m=M_{w} .
$$

The standard theory of electroweak interactions predicts that the constant of this interaction for neutral currents is the same as for charged ones:

$$
\frac{g_{z}{ }^{2}}{m_{z}{ }^{2}}=\frac{g_{w}{ }^{2}}{m_{w}{ }^{2}} .
$$

This equality shows again that all exchanged particles are formed one half-finished material. They only differ in the degree of deformation and in the number of elementary cells participating in the interaction. But since none of the physical instruments enables us to observe visually the process of particle interaction, we have to resort to probability.

The modern quantum theory that has made much progress cannot exist without the concept of "virtual" particles. The analysis made in this paper as well as in the previous and subsequent articles (ten) dealing with the theory of granular space makes us agree that the part of "virtual" objects is played by the substance (mass) carried by a bombarding particle in the form of kinetic energy and gas nothing to do with "boiling" vacuum. Weak interaction does not occur because "boiling" vacuum produced of "nothing" a virtual particle of huge mass W-boson of the instant when the particles either collide of decay thus breaking the stable low of energy conversation.

A weak reaction occurs only when even a small amount of matter (mass) transferred by a bombarding (or decaying) particle in the form of kinetic energy is compressed to the energy density equal to that of one elementary space particle.

Turning back to proton decay we wish to add the following. In modern theory a proton can decay due to the existence of heavy calibrated X-and Y-bosons.

The probability of decay is about $\alpha_{G U}^{2} m_{x}^{-4}$, where $\alpha_{G U}^{2} \approx 1 / 40$ is the constant of big unification, $m_{p}$ is the proton mass $m_{x} \sim 10^{16} \mathrm{GeV}$ in energy units (according to the present-day estimates).

The formula of proton decay probability is much the same as the formula of probability of muon decay which only occurs when the energy density of the matter under decay reaches that of a W-boson. Similarly, the energy released in proton decay must be concentrated in such a small volume that the energy density of the decaying matter $\Delta E \sim m_{p} c^{2}$ should be as high as that of a Xboson:

$$
\rho_{x} \approx \frac{m_{x} c^{2}}{\left(\hbar / m_{x} c\right)^{3}} \cong 10^{105} \mathrm{Gev} / \mathrm{cm}^{3} .
$$

If we make a fantastic that the decaying substance of the proton is concentrated in a volume of one elementary cell $\sim\left(L^{*}\right)^{3}$, its energy density will be:

$$
\rho_{p}=\frac{m_{p} c^{2}}{\left(L^{*}\right)^{3}} \cong 10^{98} \mathrm{Gev} / \mathrm{cm}^{3} .
$$

Consequently, $\rho_{p}<<\rho_{x}$ and the proton cannot decay even in the presence of $\mathrm{X}$ - and $\mathrm{Y}$-bosons.

The fact, that weak interaction is related to the energy density, directly follows from the dimension of Fermi constant:

$$
G_{F} \cong 1,4 \cdot 10^{-49} \mathrm{erg} / \mathrm{cm}^{3} \text {. }
$$

There is a wonderful analogy to this phenomenon: last energy, as usual quite low, produced a huge-power beam in a very short time and can cut a metal. Another amusing analogy is the penetration of a dense virus into the loose body of a cell. In a separate article we studied the photon motion and thus realized young Einstein's dream: what would we see if we could catch up light? Analysis of this phenomenon revealed us the pulsing character of 
proton substance motion, moving mass, and a puzzling presence of the frequency $E_{y}=\hbar \omega$ in the Planck formula. The same pulsating character enables us to observe almost visually the tunnel effect; it also explains a linear increase in the cross-section of interaction of a neutrino and an antineutrino with an electron.

We are very much surprised by the fact that the density of elementary cell matter is equal to that of a real massive W-boson. From this we can draw an amazing conclusion: nature could perfectly do without a real $\mathrm{W}$-boson and weak interaction would exist as before. It can be said that both $\mathrm{W}$ - and Z-bosons are architectural extravagances of Creator. Beside the given new glance at the role of $\mathrm{W}$-bosons in weak interaction, there is another fundamental aspect of the standard theory that seems to be artificial and vague. This is Higg's mechanism and, in particular, a Higg's boson. Nobody has any idea what Higg's sector physics consist in.

Let us turn back to the W-boson and count the number of cells whose substance is spent to form the surface layer of a particle, i.e., to form the rest mass $-N_{s}$ and the number of cells making up the "body" of W-boson $-N_{v}$. Let the particle mass be $M_{w} \approx 83 \mathrm{GeV} / \mathrm{c}^{2}$, then:

$$
\begin{gathered}
N_{s}=\frac{M_{w} c^{2}}{m_{\text {cell }} c^{2}} \approx 1,2 \cdot 10^{51} \\
N_{v}=\frac{V_{w}}{V_{\text {cell }}}=\frac{r_{w}{ }^{3}}{\left(L^{*}\right)^{3}} \approx 1,3 \cdot 10^{51}
\end{gathered}
$$

The unique coincidence between (9) and (10) means that the quantity of substance (matter) spent by space to make the material shell of a $\mathrm{W}$-boson is equal to the amount of matter inherent in the material cells making up the particle volume. None of other particles has a ratio like that, and the $\mathrm{W}$-boson goes on surprising us!

In our first paper "Granular space and the problem of big numbers" we found that if two neutral particles are tightly pressed to each other so that their surface are in contact, only the matter of one particle will take part in the gravitational interaction which reveals the secret of remarkable weakness of gravitational interaction among elementary particles. The analysis of weak interaction shows that in weak interaction, too one elementary cell comes onto the proscenium, but it is its density rather than its mass.

In addition to muon decay, now let's consider the scattering of a low-energy electron $\left(E_{e} \sim 2 \mathrm{MeV}\right)$ on a stationary proton. The cross-section of electromagnetic interaction

$\sigma_{\gamma} \sim 10^{-25} \mathrm{~cm}^{2}$, weak interaction $\sigma_{w} \sim 10^{-42} \mathrm{~cm}^{2}$. The wavelength of this electron $\lambda_{e} \sim 10^{-11} \mathrm{~cm}$. Nearly all electrons interact with a stationary proton in electromagnetic manner, and only one of $10^{17}$ electrons un- dergoes weak interaction:

$$
e^{-}+p \rightarrow n+v_{e}
$$

Which changes the quark odor: $u \rightarrow d$.

In a mental experiment, when observing the pulsing motion of an electron we can see again in what the unity of weak and electromagnetic interactions consist.

As long as the dimension of a pulsing object consisting of the mass carried by the electron kinetic energy and called today a virtual proton is comparable to $\lambda_{e}$, electromagnetic interaction occurs, and only when this object is compressed and its density is equal to that of an elementary cell and, hence, a real $\mathrm{W}$-boson, a weak reaction will take place.

That is why weak interaction becomes stronger than his more powerful brothers: electromagnetic and strong interactions. So only weak interaction can change the odors of quarks and leptons as well as break discrete symmetries: charge $-\mathrm{C}$, mirror $-\mathrm{P}$, and time $-\mathrm{T}$.

From the new viewpoint of real discrete space, this enables us to remove the accusation in breaking the law of energy conservation even for a short instant and to visualize all virtual particles which represent real objects consisting of the substance (mass) carried by kinetic energy. Later on we shall show that in statics, too, in a hydrogen atom, for example, deformed cells give birth to the same real object between the proton and the electron - a virtual photon.

And, finally, nothing prevents us from assuming that in a deuteron, too, between the proton and the neutron the deformed space cells from a more complex object simulating an exchange nuclear particle and celled at present as $\pi$-meson.

\section{Kinetic Energy and Mass}

In relativistic mechanics the kinetic energy:

$$
E_{k}=\frac{m_{0} c^{2}}{\sqrt{1-(v / c)^{2}}}-m_{0} c^{2}=\frac{m_{0}\left(1-\sqrt{1-(v / c)^{2}}\right)}{\sqrt{1-(v / c)^{2}}}=m_{k} c^{2} \text {. }
$$

Sometimes it is said that kinetic energy passes into a mass under particle collisions and vice versa. Kinetic energy needn't pass into a mass because kinetic energy is a mass or amount of matter moving ahead of the particle; this mass is designated as $m_{k}$. It is this mass that gives birth to particles during collisions. The role of space consist in the following: it lends its cells to form the particle "body" and acts as a creditor and the matter (mass) transferred by kinetic energy is used to build just the surface layer of a particle.

If vacuum is space free of physical fields, it really takes part in the formation of real particles rather than virtual ones. Besides the substance spent to form ele- 
mentary cells, vacuum has not got any additional substance which would form, at least for short instant, virtual articles, virtual in old terms. As we have already noted, vacuum only borrow its cells, for some time, to elementary particles which they must give back after annihilation. The collision of an electron with a positron ends with destruction of stable surface shells, and their substance - a rest mass - is used to form two or three photons, whereas the cells making up their body are given back to space; thus, there are no holes in space.

At present one can come across in literature the following statement: "at a high temperature $T>10^{14} \mathrm{GeV}$ all particles, including heave Higg's bosons, have no rest mass". Quite a different conclusion follows from the theory of granular space. Rest mass means the amount of substance (matter) which is spent to produce a closed surface of a particle (at least for a short instant in case of resonances) thus collectivizing the surface cells forming an original film that does not allow space to destroy the elementary particle. To remove rest mass means only one thing - to destroy the particle. To remove rest mass, sequently, even with the energy of $10^{19} \mathrm{GeV}$ all the particles retain their individuality and are not depersonalized at all.

\section{Foundation of the Calibrating Theory}

Of particular interest are local calibrating transformations which we would rather call them as phase transformations. The field theory is given by differential equations (Dirac, Klein-Gordon, etc.). Using the principle of calibration we believe that the theory must not change even when the phase depends on coordinates and time:

$$
\Psi^{\prime}(x)=e^{i \alpha(x)} \cdot \Psi(x) .
$$

It is quite evident that this transformation the derivative acquires a certain addend. The variation of the derivative with field, in its turn, would have to change the whole theory. To avoid it we must insert the compensating field $A_{\mu}$ into a theory so that it could always enter into the equation with the derivative of $\psi$ and make up a combination with it referred to as the covariant derivative:

$$
D \mu=\partial_{\mu}-i e A_{\mu}(x) .
$$

If form the very beginning it is not the ordinary derivative $\partial_{\mu}$, that enters into the theory but the covariant derivative $D_{\mu}$, this theory will be invariant about calibrated transformation:

$$
\begin{gathered}
\Psi^{\prime}(x)=e^{i \alpha(x)} \cdot \Psi(x), \\
A_{\mu}^{\prime}(x)=A_{\mu}(x)+\frac{1}{e} \partial_{\mu} \alpha(x) .
\end{gathered}
$$

This principle is a principle of relativity charge space which was first used by V. Fock in 1926.

The calibrating field $A_{\mu}$ is a physical electromagnetic field. The $e$-constant is an electric charge.

Now let us consider again an elementary cell. When studying the motion of a photon or any particle we found that it is pulsating by nature and reminds us of the motion of a lorry vibrating on a spring. The matter (mass) transferred by kinetic energy deforms the elementary cells ahead of the particle creating a real object which in the modern theory is referred to as a "virtual" photon (in electrodynamics). The pulsating character of its motion is a foundation for the dependence of the wave function phase on $x: a=a(x)$ and the virtual object (absolutely real in our theory) serves as a compensating field quan$\operatorname{tum} A_{\mu}(x)$.

Our sight keenness is not enough to observe visually both the particle and its motion. It is this circumstance that "opens the door" of probability (chance).

According to our tradition and habit, we shall use hereafter the term "virtual" though virtual particles actually consist of real elementary cells, too.

\section{Universe and an Elementary Cell}

The observable part of the Universe has dimensions $R_{u} \sim 10^{28} \mathrm{~cm}$, and knowing the mass of one elementary cell $m_{\text {cell }}$ we can easily calculate the mass of substance required for constructing material space $M_{u}$. To do this we first find the number of elementary cells filling the space of the Universe:

$$
N_{u}=\frac{4 \pi R^{3} u}{3\left(L^{*}\right)^{3}} \cong 10^{183} .
$$

From first this we get:

$$
M_{u}=N_{u} m_{\text {cell }} \cong 1,3 \cdot 10^{110} \mathrm{~g} .
$$

This figure is beyond our imagination. It is a striking fact that the Universe spent a negligible part of its substance to create elementary particles $m_{u} \sim 10^{56} \mathrm{~g}$ and the rest to form Space cells. Assuming that the Universe did not spend the whole substance to create Space and elementary particles we can propose that darkle matter and energy are the rest of this substance. The clusters of this substance do not possess either electric charges or any quantum properties and so can act on bodies only by gravitation. The mass ratio given below remains mysterious:

$$
M_{u} \cong m_{u}^{2} \text {. }
$$

Analyzing dimensions in 1900 Planck found the maximum density of matter by using only the world constants $\hbar, G$ and $c$ :

$$
\rho^{*}=\frac{3 m^{*}}{4 \pi\left(L^{*}\right)^{3}}=\frac{3 c^{5}}{4 \pi \hbar G^{2}} \cong 1,2 \cdot 10^{93} \mathrm{~g} / \mathrm{cm}^{3} \text {. }
$$


Knowing this data we can attain the dimension of extremely dense drop which was our Universe at the beginning of its development with $t=0$ :

$$
V_{u}=\frac{M_{u}}{\rho^{*}} \cong 10^{17} \mathrm{~cm}^{3} .
$$

Its initial radius $r_{u}$ and diameter $d_{u}$ are:

$$
r_{u} \cong \sqrt[3]{V_{u}} \cong 5 \mathrm{~km}, d_{u} \cong 10 \mathrm{~km} .
$$

A neutron star is almost of the same size. The result is very important for cosmology since it enables us to state that the Universe does not know the concept of singularity. The Universe has never been compressed to a point and will never become it.

Now let us study the properties of an elementary cell and determine the thickness of its walls $\Delta l_{\text {cell }}$ because we know the cell mass $m_{\text {cell }}$ and $L^{*}$ :

$$
\Delta l_{\text {cell }}=\frac{m_{\text {cell }}}{\left(L^{*}\right)^{2} \rho^{*}} \cong 10^{-100} \mathrm{~cm},
$$

and assume that the cell walls have the density of the initial drop $\rho^{*}$.

Our model allows answering some questions associated with the motion of particles in such space filled with cells.

1) The colossal density of the substance the cell walls are made of makes the cell extremely elastic and allows space to move not only photons but any other bodies with high speeds.

2) The negligible thickness of the walls enables an elementary particle, when moving, to force the space cells apart and to deform them without any resistance as the bond energy of cells is equal to zero. So space has zero the viscosity revealing the mystery of inertia.

3) This monstrous elasticity of cells is responsible for the fact large bodies (stars, galaxies), even through they are wide apart, interact with each other gravitationally; in this case close-range action passes to far-range action.

The cell turned out to be almost empty which enable the colossal mass of the Universe to shrink to a small dense lump $d \sim 10 \mathrm{~km}$.

The world constants $\hbar, G$ and $c$ enable obtaining the Planck temperature:

$$
T^{*}=\frac{1}{k}\left(\frac{\hbar c^{5}}{G}\right)^{1 / 2} \cong 1,5 \cdot 10^{32} \mathrm{~K},
$$

where $k$ is the Boltzmann constant.

Now we can outline just some preliminary considerations which, nevertheless, contain a number of important ideas about the first instant in the development of the Universe.

It is still a mystery what caused this drop to be unstable, but once it began "to boil" forming bubbles, the only thing that could occur at the moment $t>0$. Coming in contact with each other the bubbles turn to polyhedral cells thus forming Space itself. Another puzzle is what disturbed the drop of balance (initial impetus).

The space of the Universe can be only three-dimensional because it is made up of three-dimensional cells, which makes any attempts to attribute a multidimensional character to space rather pretentious. Physical theory must not only represent truthfully physical phenomena but, as opposed to pure mathematical theory, it must be constructed so that it would contain only the structure of real space and nothing more. Physics must precisely reflect the real world and suggest nothing about things not existing in reality.

The rapid boiling of a drop caused a part of the matter surrounding a group of cells formed clusters called by us as elementary particles.

This course of events excludes the probability of the Big Bang because space was born at the same time with elementary particles. The size of a drop equal to the size of a neutron star and its neutrality promt us that at the first instants only neutrons could be born; the law of barion charge conversation under such extreme conditions could hardly have worked. Neutron decay gave birth to protons, electrons and antineutrino:

$$
n \rightarrow p+e^{-}+\tilde{v}_{e} \text {. }
$$

This is a solution of the puzzle why the Universe consists only of these particles. The identity of elementary cells, in its turn, ensures the uniformity and the isotropy of space and the indistinguishability of elementary particles of the same sort, no matter at what point of the Universe they are born. Other cosmological questions will be considered in a separate article, the nature of red shift included.

We wish to understand something real and to clear up the meaning of this reality rather than just to manipulate formulas and predict correctly experimental results.

\section{Divergences in Quantum Electrodynamics}

Though the calculation algorithm of quantum-electrodynamics effect amplitudes on the basis of the diagram language is very simple and clear, it is so well defined that even computers have been used recently. Nevertheless, calculations seem to be confronted, as a rule, by insurmountable difficulties. The point is that everything is all right while we calculate the process amplitude in a first disappearing approximation of the perturbation theory, but when we begin higherapproximation calculations, the scattering amplitude develops divergent integrals. Divergences occur in the range of large momenta of virtual particles. 
The calculations of the Lamb shift and the abnormal electron magnetic moment will have an intelligent form if we introduce integral cutting and consider the virtual photon momentum not to exceed a certain ultimate momentum $L$.

A poor result of cutting will be, of course, the relativistic invariance of theory. For a long time these problems prevented further advance. But the introduction of the Fermi momentum cannot solve the problem of divergences yet. The procedure of divergent integral cutting must be supplemented with the idea of electron mass and charge renormalization. In this case from the expressions derived we subtract several terms which are in general infinitely large. In this connection Dirac said: “...the calculation procedure, with infinities neglected, is mathematically quite unacceptable. In mathematics a quantity is neglected only if it is very small but not because it is infinitely large and we want to get rid of it! But, due to a good correlation with experiment we begin to suspect that there is something truthful in this procedure. The task is to modify the subtraction procedure so that it could be logical, with the obtained good results retained" [10].

Now let us consider the new thing introduced by the granular space theory into this problem. In this case it should be reminded that in forming an elementary particle in space filled with elementary cells the additional substance (particle mass) is spent to form only the particle surface since its "body" is made of space cells. The particle mass has to deform both the inner and the outer cells of the "body".

It is obvious that a certain part of space cells will be pushed out and make up reserve of matter which the space can transfer to the particles thus realizing interactions. We call this reserve as the potential energy and in this way unravel the secret: where space gets this energy from. This additional mass is precisely equal to the mass of the new-born particle - a peculiar Archimedes law in microphysics. The deformation of these outer layers around the particle forms a field brilliantly developed then, starting from the fundamental works of Faraday and Maxwell.

Let's turn to the proton-electron system where the electron is far from proton. The deformation of the cells, owing to the difference is charge signs, will be larger from the external side of the electron than from the inner side and the space pushes the electron towards the proton, so we can observe this process almost visually. Space in this process plays the role of a creditor rather than a usurer because it does not take any interest from "debtor". In other words, a part of matter from the reserve, resulting by the formation of particles, is transferred to the electron by space - the process called the transition of poten- tial energy to kinetic one.

Assume that the electron transfers to the second energy level. According to the virial theorem, the electron releases exactly half its energy obtained from the field in the form of a photon and leaves the second half for itself. Thus, staying on the second level for a while the electron at the same time possesses a kinetic energy, a momentum and father a considerable speed. This paradox can be easily resolved if we recall that the theory of granular space reveals the physical meaning of velocity and acceleration: velocity is not only a characteristic of speed of displacement in the given system of reference but, to a greater extent, a characteristic of deformation of space elementary cells: $\varepsilon=v^{2} / c^{2}$ is the relative deformation and acceleration is the gradient of this deformation. Fifty years ago J. Zeldovich was right saying that the tensors entering into Einstein's gravity equation describe the elasticity of space. If we had keener eyesight we could see that the matter related to kinetic energy, as it deforms the elementary cells between the proton and the electron, forms the "body" of an object called in the modern theory as the "virtual" photon. There is no throw-over from the proton to the electron, or volleyball game, here and not the slightest hint of vacuum "boiling" and the creation of quite an army of virtual particles. As before, we shall call our real object as the virtual photon so as not to change the accepted terminology.

To calculate precisely the Lamb shift and the abnormal magnetic momentum of electron and muon we should consider at present more than 800 Feynman's diagrams using in this case numerous virtual particles.

Our studies into the discrete structure of material space inevitably make us acknowledge that all the processes occurring in the Universe can be reduced to one of them - the deformation of elementary cells. Consequently, the higher - order consideration of the theory of interaction of various loop diagrams allows us to account more precisely for the peculiarities of this deformation in calculating the Lamb shift and the abnormal magnetic momenta of electron and muon:

Taking the second-order polarization operator as an example $\Pi_{\mu \nu}{ }^{2}(k)$, let's consider the new thing introduced into its calculation by the theory of granular space.

This quantity (Figure 4(b)) is determined, according to the Feynman rules, by the formula [4]:

$$
\Pi_{\mu \nu}{ }^{2}(k)=\frac{e^{2}}{(2 \pi)^{4}} S p \int \gamma_{\mu} \frac{i p-m}{p^{2}+m^{2}} \cdot \gamma_{v} \frac{i(p-k)-m}{(p-k)^{2}+m^{2}} \cdot d^{4} p
$$

To calculate $\Pi_{\mu v}{ }^{2}(k)$, we must perform rather cumbersome manipulations. As a result, we have:

$$
\Pi_{\mu \nu}{ }^{2}(k)=\frac{i e^{2}}{(2 \pi)^{2}}\left\{\frac{L^{2}-m^{2}}{2}+\frac{k^{2}}{3}\left(\frac{5}{6}-\ln \frac{L^{2}}{m^{2}}\right)+\right.
$$



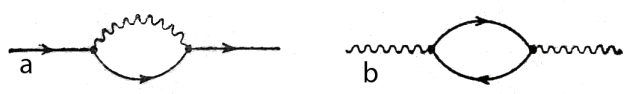

Figure 4. Electromagnetic mass of electron (a), vacuum polarization (b).

$$
\left.+\int_{0}^{1} d x\left[\frac{1}{2} k^{2}-m^{2}-k^{2} x(1-x) \cdot \ln \left[1+\frac{k^{2}}{m^{2}} \cdot x(1-x)\right]\right]\right\}
$$

$L$ is the boundary momentum $k$ is the momentum of virtual exchange photon that characterizes the deformation of cells between the proton and the electron. The kinetic energy of an electron on the second energy level of hydrogen atom is rather low, $E \sim 3,6 \mathrm{eV}$; The energy of the Lamb shift - the transition of an electron from the level ${ }^{2} S_{1 / 2}$ to ${ }^{2} P_{1 / 2}$ - is much lower:

$$
\Delta E \sim 4,6 \cdot 10^{-6} \mathrm{eV} .
$$

The situation reminds of a weak interaction between an electron and a proton discussed above. Despite the low energy of a bombarding electron $E \sim 2 \mathrm{MeV}$, the $\mathrm{W}$-boson density entering into the formula is huge and equal the elementary cell density. But this density was needed to "unseal" the surface of the proton and to change the quark's color.

Similarly, in the Lamb shift the transition of the electron to a lower level requires the maximum density of virtual photon matter which is related to the electron mass because the highest energy in this process is the electron rest energy $E=0,511 \mathrm{MeV}$; the proton can be considered absolutely stationary.

We have to say that the squared momentum circulating along a loop $p^{2}$ cannot be more than the electron squared mass (in energy units) $p^{2}=m_{e}{ }^{2}$ which means, in its turn, that the boundary momentum $L^{2}=m_{e}{ }^{2}$.

This unique conclusion of the theory of granular space not only retains the invariance of integral cutting but also reduced to zero the former infinite values since, with $L^{2}=m_{e}^{2}$, we have:

$$
L^{2}-m_{e}^{2}=0 \text { and } \ln \left(L^{2} / m_{e}^{2}\right)=\ln 1=0 .
$$

And only now it becomes clear why the cancelling of the infinitely large terms from the formulas resulted in a good agreement between theory and experiment: we cancelled the zeroth-order terms and not the infinite ones! This comment refers to the calculation of both the third-order peak function $\Lambda_{\mu}{ }^{3}(p 1, p 2)$ and the mass operator $\sum^{2}(p)$.

All the above-said makes us reconsider completely the procedure of renormalizing both the charge and the electron mass because they don't gain any infinite additions $\delta m$ and $\delta e$ in physical processes.
The renormalizing procedure has been described in a lot of monographs, and here we just touch upon this operation.

Let us denote the electron charge appearing in Feynman lowest-order diagrams by $e$, the electric charge given in the tables of elementary particle properties by $e_{R}$, i.e.:

$$
e_{R}^{2} / \hbar c=1 / 137,0359 \text {. }
$$

To renormalize the charge we introduce the relation:

$$
e_{R}=e\left(1-\frac{e^{2}}{12 \pi^{2}} \ln \frac{L^{2}}{m^{2}}\right)^{1 / 2}
$$

where $L$ is limiting momentum circulating along the loop in calculating, for example, the Rutherford scattering amplitude. The infinity related to the transition to an infinite limit of the cutting parameter $(L \rightarrow \infty)$ is "absorbed" in $e_{R}$. This procedure seems really strange but, according to our statement, $L^{2}=m^{2}$ and $e_{R}=e$.

Dirac wrote: “... physicists are mistaken trying repeatedly to develop physical ideas they have got used to: these are ideas usually expressed in the language of Feynman diagrams. We should not adhere obstinately to habitual ideas trying to exploit them".

Using the granular structure of space we can assert that, as the electron passes from the level ${ }^{2} P_{1 / 2}$ to ${ }^{2} S_{1 / 2}$ in a hydrogen atom, $10^{50}$ elementary cells of space participate in this process almost simultaneously. As Feynman aptly expressed, we are to guess right what happens to the cells during their collectivization or clusterization but now we know a physical process - light diffraction we can make start from.

To understand the essence of the method developed by Frenel, let's determine the amplitude of the light oscillation excited at the point $\mathrm{P}$ by a spherical wave propagating in isotropic uniform medium from a point source $S$. The wave surface in this case is symmetric about the straight line SP. In view of the fact, we divide the wave surface into ring zones so that the distances from the edges of every zone to the point $\mathrm{P}$ differ by $\lambda / 2$. They are called as Frenel zones.

The amplitudes of the oscillations excited at the point $\mathrm{P}$ by Frenel zones from a monotonically decreasing sequence:

$$
A_{1}>A_{2}>A_{3} \ldots \ldots
$$

The phases of the oscillations excited by adjacent zones differ by $\pi$. Therefore the amplitude A of the resulting oscillation at the point $\mathrm{P}$ can be expressed as

$$
A=A_{1}-A_{2}+A_{3}-A_{4} \ldots \ldots
$$

Now let us calculate the abnormal magnetic momentum of electron $\mu_{e}$. Summing up all the contributions into $\mu_{e}$, up to the third order of perturbation theory, we get a theoretical value: 


$$
\frac{\mu_{\text {teor }}}{\mu_{0}}=1+\frac{\alpha}{2 \pi}-1,31392 \cdot\left(\frac{\alpha}{2 \pi}\right)^{2}+9,464\left(\frac{\alpha}{2 \pi}\right)^{3}-\ldots \ldots . .
$$

where $\mu_{0}=e \hbar / 2 m_{e} c$ is Bohr magneton.

This formula closely resembles the summation formula of Frenel amplitudes. We specially separate out the expression $a / 2 \pi$ the inverse of which, with high precision, equals 861 .

The surprising thing is that all the factors in front of $(a / 2 \pi)^{n}$ are about 1 despite all the huge attempts made by theorists to estimate them. A high accuracy can be gained with $a / 2 \pi \cdot 10^{-3}$, the value by which every subsequent term in (10) is reduced, and this is a real gift of Nature.

It is quite possible that the above-said real object (our virtual photon) positioned between the electron and the proton has a surface with the same ring-shaped structure the Frenel surface has.

In 1891 G. Cantor proposed a theorem about the impossibility of counting real number multitudes thus trying to actualize infinity and work with it like with standard finite numbers. This free treatment with infinity cause sharp protest of many famous mathematicians. "There is not actual infinity. Cantorians forgot it and gave way to contradictions. They fell into excessive generalization and forgot that freedom is not an arbitrary rule", wrote A. Puancare.

Nature, however, knows only finite natural numbers and, therefore, there cannot be infinity in studying physical processes. Only this should have banned infinite limits in integral calculations.

Physical theory must not only represent physical phenomena correctly but also, in contrast to purely mathematical theory, must be worked out so that it would contain only the structure of real space and nothing else. Physics must truthfully reflect the real world and not suggest anything about things which do not exist in reality. Since the electron mass $m_{e}$ is the largest real (not virtual, mental) amount of substance, matter at our disposal in studying the Lamb shift, the integration limits cannot exceed $m_{e}$.

\section{Conclusions}

The assumption of the material granular structure of space enables visualizing quite a number of physical processes and phenomena:

1) The pulsating character of motion of both: a photon and any elementary particle.

2) All virtual particles are material objects consisting of deformed elementary cells of space.

3) The elementary cell mass is determined by two independent ways.

4) The absolute stability of proton has been proved.

5) The energy density of W-boson is equal to the elementary cell density.

6) Infinite integrals arising in the renormalization procedure are equal to " 0 " and the procedure itself takes a relativistically invariant form.

\section{References}

[1] M. Planck, "The Unity of the Physical Pattern of the World," NAUKA, Moscow, 1966.

[2] R. Feynman, "The Feynman Lectures on Physics," Addison-Wesley Publishing Company, London, 1963.

[3] V. Konushko, “Concept Granular Space Theory," SPUTNIC, Moscow, 1999.

[4] Wheeler, "Einstein Visions," Springler-Verlag, New York, 1968.

[5] G. Jacobson and R. Parentani, "The Echo of the Black Holes," Scientific American, No. 3, 2006, p. 17.

[6] L. Smolin, “Atom s Space and Time," Scientific American, No. 4, 2004, p. 20.

[7] L. Brillouin, "Relativity Reexamined," Academic Press, New York and London, 1970.

[8] S. Weinberg, "Recent Progress in the Gauge Theories of the Weak, Electromagnetic and Strong Interactions," Reviews of Modern Physics, Vol. 46, No. 2, 1974, pp. 255277. doi:10.1103/RevModPhys.46.255

[9] Salam, "Unification of Fundamental Forces," Cambridge University Press, Cambridge, 1990.

[10] P. A. M. Dirac, "Reminiscences of the Unusual Epoch," NAUKA, Moscow, 1990. 